\title{
EXTREMAL UNICYCLIC GRAPHS WITH RESPECT TO ADDITIVELY WEIGHTED HARARY INDEX
}

\author{
JELENA SEDLAR
}

Received 24 September, 2013

\begin{abstract}
In this paper we define cycle-star graph $C S_{k, n-k}$ to be a graph on $n$ vertices consisting of the cycle of length $k$ and $n-k$ leafs appended to the same vertex of the cycle. Also, we define cycle-path graph $C P_{k, n-k}$ to be a graph on $n$ vertices consisting of the cycle of length $k$ and of path on $n-k$ vertices whose one end is linked to a vertex on a cycle. We establish that cyclestar graph $C S_{3, n-3}$ is the only maximal graph with respect to additively weighted Harary index among all unicyclic graphs on $n$ vertices, while cycle-path graph $C_{3, n-3}$ is the only minimal unicyclic graph (here $n$ must be at least 5). The values of additively weighted Harary index for extremal unicyclic graphs are established, so these values are the upper and the lower bound for the value of additively weighted Harary index on the class of unicyclic graphs on $n$ vertices.
\end{abstract}

2010 Mathematics Subject Classification: 05C35

Keywords: additively weighted Harrary index, unicyclic graph, extremal graph

\section{INTRODUCTION}

A topological index of a graph is a number attributed to a graph in a way that it is derived from the structure of the graph but so that it doesn't depend on the labeling of vertices in a graph. Chemical graph theory is a branch of graph theory whose focus of interest is finding topological indices of chemical graphs (i.e. graphs that represent chemical molecules) which correlate well with chemical properties of the corresponding molecules. One of the most famous topological indices is Wiener index, defined as the sum of all distances between different vertices of a molecular graph, introduced by Wiener in 1947 (see [10]) in a paper concerned with boiling points of alkanes. In a research that followed many other useful properties of Wiener index were discovered (for a survey of mathematical properties and chemical applications of Wiener index one can look into [3,4,7]). But, contrary to chemical intuition, the contribution of close pairs of vertices to the overall value of the index was much smaller than that of distant vertices. To deal with this inconsistency, the new index was proposed which was named Harary index $([6,8])$. Harary index is defined as the

The support of EUROCORES Programme EUROGIGA (project GReGAS) of the European Science Foundation and Croatian Science Foundation under the project 8481 is gratefully aknowledged. 
sum of all reciprocal values of distances between different vertices of a molecular graph. Properties of Harary index were then extensively researched (see for example $[5,9,11,12])$. But it turned out that this modification of Wiener index has not solved the inconsistency as expected. In order to improve the performance of Harary-type indices, several modifications were proposed recently. In [2] the authors increased the attenuation of contributions of vertex pairs with their distance. In [1] an attempt was made in a different direction, the authors introduced a correction that gives more weight to the contributions of pairs of vertices of high degrees. This modification was named additively weighted Harary index. The aim of this paper is to establish the upper and lower bound for the value of additively weighted Harary index on the class of unicyclic graphs and to characterize all extremal graphs in that class.

The present paper is organized as follows. In Section 2 we introduce necessary definitions and preliminary results. In Section 3 we characterize all maximal unicyclic graphs by introducing several graph transformations which increase the value of additively weighted Harary index and which, when applied combined finitely many times, lead to maximal unicyclic graphs. In Section 4 we do the same for minimal unicyclic graphs. Finally, in Section 5 we give the conclusion and directions for further research. The paper is completed with acknowledgements and references.

\section{PRELIMINARIES}

Let $G=(V, E)$ be a graph with set of vertices $V$ and set of edges $E$. In this paper all graphs are finite, simple and connected. For a pair of vertices $u, v \in V$ the distance $d_{G}(u, v)$ is defined as the length of the shortest path between $u$ and $v$. The degree $\delta_{G}(v)$ of a vertex $v \in V$ is defined as the number of vertices in $V$ neighboring to $v$. A leaf in a graph $G$ is every vertex in $G$ of degree 1 . Additively weighted Harary index of a graph $G$ is defined as

$$
H_{A}(G)=\sum_{u \neq v} \frac{\delta_{G}(u)+\delta_{G}(v)}{d_{G}(u, v)} .
$$

We say that graph $G$ is a tree if $G$ doesn't contain a cycle. We say that $G$ is a unicyclic graph if $G$ contains exactly one cycle. The set of all unicyclic graphs on $n$ vertices will be denoted by $U(n)$. The only cycle in a unicyclic graph $G$ will be denoted by $C$ and the length of $C$ will be denoted by $c$. Usually, we will suppose $C$ consists of path $P=w_{i} w_{i+1} \ldots w_{i+c-1}$ and an edge $w_{i} w_{i+c-1}$. Starting index $i$ will not always be the same. Vertex $w_{i-j}$ will denote $j$-th vertex on $C$ from $w_{j}$ in negative direction, while $w_{i+j}$ will denote $j$-th vertex on $C$ from $w_{j}$ in positive direction. Note that in this way we possibly introduce alternative labels of vertices on $C$. For example, if $C=w_{0} w_{1} \ldots w_{5}$, then $w_{-2}=w_{4}$ and $w_{7}=w_{1}$. In other words, indices are added modulo $c$. We say $v \in G \backslash C$ is branching if $\delta_{G}(v) \geq 2$, we say $v \in C$ is branching if $\delta_{G}(v) \geq 3$. 
A cycle-star graph is a unicyclic graph consisting only of a cycle and leafs appended to vertices of the cycle. Note that a cycle-star graph can be obtained from stars and a cycle by identifying central vertex of stars with different vertices on the cycle, hence the name. A cycle-path graph is a unicyclic graph consisting only of a cycle and at most one path appended to each vertex of the cycle. We denote by $C S_{k, n-k}$ a cycle-star graph on $n$ vertices consisting of the cycle of length $k$ and $n-k$ leafs appended to the same vertex of the cycle. We denote by $C P_{k, n-k}$ a cycle-path graph on $n$ vertices consisting of the cycle of length $k$ and the path on $n-k$ vertices whose end vertex is linked to a vertex on the cycle. These notions are illustrated in Figures 1 and 2.

a)

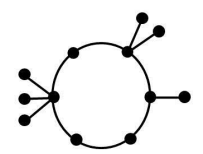

b)

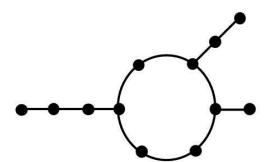

FigURE 1. An example of: a) cycle-star, b) cycle path.

a)

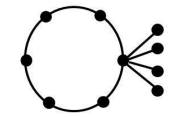

b)

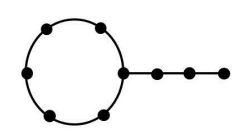

FiguRE 2. Graphs: a) $C S_{6.4}$, b) $C P_{6,3}$.

The cycle on $n$ vertices will be denoted by $C_{n}$. Obviously, it holds that $C_{n} \in \mathcal{U}(n)$. It is also convenient to consider that $C_{n}$ is both a cycle-star and a cycle-path graph, i.e. $C_{n}=C S_{n, 0}=C P_{n, 0}$.

Finally, we have to introduce numbers which are quite useful when expressing the values of Harary index. Harmonic number $H_{n}$, where $n \geq 1$ is an integer, is defined as $H_{n}=\sum_{i=1}^{n} \frac{1}{i}$. We define also $H_{0}=0$.

Before we proceed to our main results, we will prove two simple lemmas which will be useful later.

Lemma 1. For $n \geq 3$ it holds that:

(1) $H_{A}\left(C S_{3, n-3}\right)=\frac{3}{2}\left(n^{2}-n+2\right)$,

(2) $H_{A}\left(C P_{3, n-3}\right)=4 \sum_{i=1}^{n-2} H_{n-i-1}+H_{n-3}+3 H_{n-2}+\frac{6 n-13}{n-2}$.

Proof. By direct calculation.

Also, we introduce the following lemma, which is the direct consequence of the fact that $n$-th Harmonic number $H_{n}$ is roughly equal to the natural logarithm of $n$. 
Lemma 2. For odd $n \geq 5$ it holds that $\frac{3}{2}\left(n^{2}-n+2\right)-4 n \cdot H_{\frac{n-1}{2}}>0$.

\section{MAXIMAL UNICYCLIC GRAPHS}

We will find maximal unicyclic graphs by introducing transformations of a graph $G$ to $G^{\prime}$ which increase the value of $H_{A}$, therefore $H_{A}\left(G^{\prime}\right)-H_{A}(G)>0$. Since $H_{A}\left(G^{\prime}\right)-H_{A}(G)$ is a sum over all pairs of vertices in $G$, it is very convenient to denote by $\Delta(u, v)$ the contribution of a pair $u, v \in G$ to the sum $H_{A}\left(G^{\prime}\right)-H_{A}(G)$. The problem to solve will be negative contributions of certain pairs of vertices, for which we will have to find enough pairs with positive contribution to compensate in the sum.

Lemma 3. For an odd $n \geq 5$ it holds that $H_{A}\left(C_{n}\right)<H_{A}\left(C S_{3, n-3}\right)$.

Proof. For an odd $n$, it is easily verified that $H_{A}\left(C_{n}\right)=4 n \cdot H_{\frac{n-1}{2}}$. From Lemma 1 we have $H_{A}\left(C S_{3, n-3}\right)=\frac{3}{2}\left(n^{2}-n+2\right)$. Now the claim follows from Lemma 2.

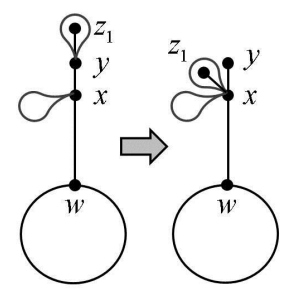

FIGURE 3. Graph transformation from the proof of Lemma 4.

Lemma 4. For every $G \in \mathcal{U}(n)$ which is not a cycle-star graph, there is a cyclestar graph $G^{\prime} \in U(n)$ such that $H_{A}(G)<H_{A}\left(G^{\prime}\right)$.

Proof. Let $C$ be the only cycle in $G$ and let $w \in C$ be the branching vertex. Let $T$ be the connected component of $G \backslash C$ containing $w$. Note that $T$ must be a tree. Let $z_{1}$ be a leaf in $T$ furthest from $w$. Let $P=w \ldots x y z_{1}$ be the shortest path between $w$ and $z_{1}$. Let $z_{1}, z_{2}, \ldots, z_{k}$ be all leafs neighboring to $y$. Since $z_{1}$ is a leaf furthest from $w$, it follows that $k=\delta_{G}(y)-1$. Let $G^{\prime}$ be a graph obtained from $G$ by deleting edges $z_{i} y$ for $i=1, \ldots, k$ and adding edges $z_{i} x$ instead. This transformation is illustrated in Figure 3. We have to prove that $H_{A}(G)<H_{A}\left(G^{\prime}\right)$. Because of the definition of the index $H_{A}$, the problem are pairs of vertices whose distance increases and vertices whose degree decreases. Note that in this transformation the only pairs $u, v$ whose distance increases are pairs $u=y$ and $v=z_{i}$. Also, the only vertex for which degree decreases is $y$. Therefore, contributions $\Delta\left(y, z_{i}\right)$ will be negative due to increase in distance, while contributions $\Delta(y, v)$, where $v \in G$, will (possibly) be negative due 
to decrease in degree of vertex $y$. Note that contributions $\Delta(y, v)$ are not necessarily negative, since degree of $v$ can increase or the distance $d_{G}(y, v)$ can also decrease.

Let us first consider the problem with the increase in distance. We will show that the negative contribution $\Delta\left(y, z_{i}\right)$ is compensated by the positive contribution $\Delta\left(x, z_{i}\right)$. More formally, we have

$$
\begin{aligned}
\Delta\left(y, z_{i}\right)+\Delta\left(x, z_{i}\right) & =\frac{1+1}{2}-\frac{1+k+1}{1}+\frac{\delta_{G}(x)+k+1}{1}-\frac{\delta_{G}(x)+1}{2} \\
& =\frac{\delta_{G}(x)-1}{1}-\frac{\delta_{G}(x)-1}{2}>0 .
\end{aligned}
$$

Let us now consider the problem with degrees. We have to consider contributions $\Delta(y, v)$, where $v \in G$. First, note that we have already considered and compensated contributions $\Delta\left(y, z_{i}\right)$. Further, note that not all of these contributions are necessarily negative, since there can be increase in degree of the other vertex. So, for $v=x$ we have

$$
\Delta(y, x)=\frac{1+(k+\delta(x))}{1}-\frac{(k+1)+\delta_{G}(x)}{1}=0 .
$$

For $v \in G \backslash\left\{x, y, z_{1}, \ldots, z_{k}\right\}$, negative contribution $\Delta(y, v)$ can be compensated by the positive contribution $\Delta(x, v)$. More formally, we have

$$
\begin{aligned}
\Delta(y, v)+\Delta(x, v)= & \frac{1+\delta_{G}(x)}{d_{G}(y, v)}-\frac{k+1+\delta_{G}(x)}{d_{G}(y, v)} \\
& +\frac{k+\delta_{G}(x)+\delta_{G}(v)}{d_{G}(y, v)-1}-\frac{\delta_{G}(x)+\delta_{G}(v)}{d_{G}(y, v)-1} \\
= & \frac{k}{d_{G}(y, v)-1}-\frac{k}{d_{G}(y, v)}>0 .
\end{aligned}
$$

Therefore, we have proved $H_{A}(G)<H_{A}\left(G^{\prime}\right)$. If $G^{\prime}$ is a cycle-star graph, then the proof is over, else this transformation must be repeated finitely many times in order to obtain a cycle-star graph and then the proof is over.

a)

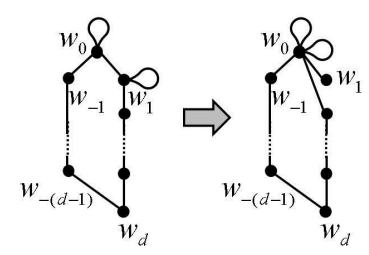

b)

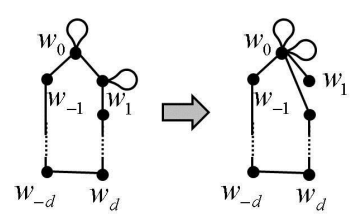

FIGURE 4. Graph transformation from the proof of Lemma 5: a) even $c, \mathrm{~b}$ ) odd $c$. 
Lemma 5. Let $G \in U(n)$ be a cycle-star graph with the length of the only cycle being $c \geq 4$. Then there is a cycle-star graph $G^{\prime} \in \mathcal{U}(n)$ with the length of the only cycle being 3 for which $H_{A}(G)<H_{A}\left(G^{\prime}\right)$.

Proof. Let $C$ be the only cycle in $G$ and let $c$ be the length of $C$. Let $d=\lfloor c / 2\rfloor$. In the case of even $c$ we will denote vertices on $C$ by $C=w_{-(d-1)} \ldots w_{d}$, in the case of odd $c$ we will denote $C=w_{-d} \ldots w_{d}$. Without loss of generality we may assume that $w_{-d}$ (if it exists, since it exists only for odd $c$ ) is of minimum degree among vertices on $C$. It is convenient to introduce the notation $k_{i}=\delta_{G}\left(w_{i}\right)$. Now, leafs neighboring to $w_{i}$ will be denoted by $x_{i, j}\left(j=1, \ldots, k_{i}-2\right)$ and we define $V_{i}=\left\{w_{i}, x_{i, j}: j=1, \ldots, k_{i}-2\right\}$. Also, let $V^{+}=\left(V_{1} \cup \ldots \cup V_{d}\right) \backslash\left\{w_{1}\right\}$ and $V^{-}=\left(V_{0} \cup \ldots \cup V_{-(d-1)}\right) \backslash\left\{w_{0}\right\}$. Obviously, in the case of even $c$ it holds that $V=\left\{w_{0}, w_{1}\right\} \cup V^{+} \cup V^{-}$, while in the case of odd $c$ it holds that $V=\left\{w_{0}, w_{1}\right\} \cup$ $V^{+} \cup V^{-} \cup V_{-d}$.

Now, let $G^{\prime}$ be a graph obtained from $G$ by deleting all edges $v w_{1}$ incident to $w_{1}$, except $w_{0} w_{1}$, and adding the edge $v w_{0}$ instead. This transformation is illustrated in Figure 4. Obviously, $G^{\prime}$ is a cycle-star graph in which the only cycle is of the length $c-1$. We have to prove that $H_{A}(G)<H_{A}\left(G^{\prime}\right)$. Note that in this transformation the only pairs $u, v$ whose distance increases are pairs $u=w_{1}$ and $v \in V^{+} \cup V_{-d}$ (recall that $V_{-d}$ only exists for odd $c$ ). Also, the only vertex whose degree decreases is $w_{1}$. Therefore, we have to consider all contributions $\Delta\left(w_{1}, v\right)$ where $v \in G$.

If $v=w_{0}$ we have $\Delta\left(w_{1}, w_{0}\right)=0$, else if $v \in V^{+}$we have

$$
\Delta\left(w_{1}, v\right)+\Delta\left(w_{0}, v\right)=\frac{k_{0}-1}{d_{G}\left(w_{1}, v\right)}-\frac{k_{0}-1}{d_{G}\left(w_{1}, v\right)+1}>0,
$$

else if $v \in V^{-}$we have

$$
\Delta\left(w_{1}, v\right)+\Delta\left(w_{0}, v\right)=\frac{k_{1}-1}{d_{G}\left(w_{1}, v\right)-1}-\frac{k_{1}-1}{d_{G}\left(w_{1}, v\right)}>0 .
$$

Note that in the case of even $c$ this completes the proof. In the case of odd $c$ we still have to consider contributions $\Delta\left(w_{1}, v\right)$ where $v \in V_{-d}$. First, note that for $G=C_{n}$ the claim of this lemma follows from Lemma 3. So, we will suppose $G \neq C_{n}$. Now, for negative contributions $\Delta\left(w_{1}, v\right)$, where $v \in V_{-d}$, positive contributions $\Delta\left(w_{0}, v\right)$ will not suffice to compensate, so we will have to find more pairs with positive contribution for compensation. To prove this more formally, we will distinguish cases $v=w_{-d} \in V_{-d}$ and $v=x_{-d, j} \in V_{-d}$.

If $v=w_{-d} \in V_{-d}$ we have

$$
\Delta\left(w_{1}, w_{-d}\right)+\Delta\left(w_{0}, w_{-d}\right)=\frac{1+k_{-d}}{d+1}-\frac{1+k_{-d}}{d}<0 .
$$

Therefore, we have to find more pairs with positive contributions to compensate. Since $G \neq C_{n}$ there has to be at least one leaf $x_{i, 1}$ in $G$. Without loss of generality 
we can assume $i \leq 0$. Then for $x_{i, 1}$ and $w_{j}$ where $j=d-i$, recalling that $w_{-d}$ is of minimum degree on $C$ (i.e. $k_{j} \geq k_{-d}$ ), we have

$$
\Delta\left(x_{i, 1}, w_{j}\right)=\frac{1+k_{j}}{d}-\frac{1+k_{j}}{d+1} \geq\left[k_{j} \geq k_{-d}\right] \geq \frac{1+k_{-d}}{d}-\frac{1+k_{-d}}{d+1}>0 .
$$

So, obviously $\Delta\left(w_{1}, w_{-d}\right)+\Delta\left(w_{0}, w_{-d}\right)+\Delta\left(x_{i, 1}, w_{j}\right) \geq 0$.

Finally, if $v=x_{-d, j} \in V_{-d}$ we have

$$
\Delta\left(w_{1}, x_{-d, i}\right)+\Delta\left(w_{0}, x_{-d, i}\right)=\frac{2}{d+2}-\frac{2}{d+1}<0 .
$$

Again, it follows that we have to find more pairs with positive contribution to compensate. Since $w_{-d}$ is of minimum degree on $C$, it follows that every $w_{i}$ has at least as many leafs as $w_{-d}$. Let us now consider pair of leafs $x_{0,1}$ and $x_{d, i}$. We have

$$
\Delta\left(x_{0,1}, x_{d, i}\right)=\frac{2}{d+1}-\frac{2}{d+2}>0 .
$$

Obviously, we have $\Delta\left(w_{1}, x_{-d, i}\right)+\Delta\left(w_{0}, x_{-d, i}\right)+\Delta\left(x_{0,1}, x_{d, i}\right)=0$ which completes the proof.

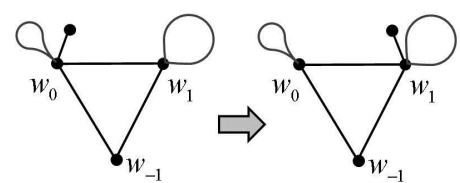

FIGURE 5. Graph transformation from the proof of Lemma 6.

Lemma 6. Let $G \in \mathcal{U}(n)$, where $G \neq C S_{3, n-3}$, be a cycle-star graph with the length of the only cycle being $c=3$. Then $H_{A}(G)<H_{A}\left(C S_{3, n-3}\right)$.

Proof. Let $C=w_{-1} w_{0} w_{1}$ be a cycle in $G$ and let $k_{i}=\delta_{G}\left(w_{i}\right)$. Let $x_{i, j}$ be all leafs attached to $w_{i}$ and let $V_{i}=\left\{w_{i}, x_{i, j}: j=1, \ldots, k_{i}-2\right\}$. Since $G \neq C S_{3, n-3}$, it follows that at least two vertices on $C$ are branching. Without loss of generality we can suppose that $w_{0}$ has minimum degree and $w_{1}$ maximum degree among branching vertices on $C$. Let $G^{\prime}$ be a graph obtained from $G$ by deleting the edge $x_{0,1} w_{0}$ and adding the edge $x_{0,1} w_{1}$ instead. We have to prove $H_{A}(G)<H_{A}\left(G^{\prime}\right)$.

Note that in this transformation the only pairs $u, v$ whose distance increases are pairs $u=x_{0,1}$ and $v \in V_{0}$. The only vertex whose degree decreases is $w_{0}$. Let us first consider the problem with the increase in distances. It is easily verified that $\Delta\left(x_{0,1}, x_{0, j}\right)+\Delta\left(x_{0,1}, x_{1, j}\right)=0$. Also, we have

$$
\Delta\left(x_{0,1}, w_{0}\right)+\Delta\left(x_{0,1}, w_{1}\right)=\frac{k_{1}+1-k_{0}}{1}-\frac{k_{1}+1-k_{0}}{2}>\left[k_{1} \geq k_{0}\right]>0 .
$$


Let us now consider the problem with the increase in degree. It is easily verified that for $v \in V_{-1}$ we have $\Delta\left(w_{0}, v\right)+\Delta\left(w_{1}, v\right)=0$ and also that $\Delta\left(w_{0}, w_{1}\right)=0$. Further, we have

$$
\begin{gathered}
\Delta\left(w_{0}, x_{0, j \geq 2}\right)+\Delta\left(w_{1}, x_{0, j \geq 2}\right)=-\frac{1}{2}<0 . \\
\Delta\left(w_{0}, x_{1, j}\right)+\Delta\left(w_{1}, x_{1, j}\right)=\frac{1}{2}>0 .
\end{gathered}
$$

Note that the negative contribution $\Delta\left(w_{0}, x_{0, j \geq 2}\right)$ is still not compensated, since the positive contribution $\Delta\left(w_{1}, x_{0, j \geq 2}\right)$ is not enough. But since $k_{0} \leq k_{1}$, it follows that for each $x_{0, j}$ there is the corresponding $x_{1, j}$. So, for $j=2, \ldots, k_{0-2}$ we have

$$
\Delta\left(w_{0}, x_{0, j}\right)+\Delta\left(w_{1}, x_{0, j}\right)+\Delta\left(w_{0}, x_{1, j}\right)+\Delta\left(w_{1}, x_{1, j}\right)=0,
$$

which means we have finally compensated for the negative contribution $\Delta\left(w_{0}, x_{0, j \geq 2}\right)$. By applying this transformation finitely many times, we will obtain the desired graph $C S_{3, n-3}$, so the proof is over.

Theorem 1. Let $G \in \mathcal{U}(n)$. Then

$$
H_{A}(G) \leq \frac{3}{2}\left(n^{2}-n+2\right)
$$

with equality if and only if $G=C S_{3, n-3}$.

Proof. Using Lemmas 4, 5 and 6 we first transform $G$ to a cycle-star graph, then we reduce the length of the cycle to 3 , so that we can finally transform it to $C_{3, n-3}$. In each transformation the value of $H_{A}$ increases, so $G=C_{3, n-3}$ is the only maximal unicyclic graph. Note that the case of $G=C_{n}$ is covered by Lemma 3 for $n$ odd, while for even $n$ it is covered by Lemma 5. Now the bound for $H_{A}$ follows from Lemma 1.

\section{Minimal UNICYCLIC GRAPHS}

As in previous section, we will find minimal graphs by introducing transformations of a graph $G$ to $G^{\prime}$, but which now decrease the value of $H_{A}$. Therefore, in this section $\Delta(u, v)$ will denote the contribution of a pair of vertices $u, v \in G$ to the sum $H_{A}(G)-H_{A}\left(G^{\prime}\right)$. The problem will again be pairs of vertices with negative contribution, for which we will have to find enough pairs with positive contribution to compensate in the sum.

Lemma 7. For every $G \in \mathcal{U}(n)$ which is not a cycle-path graph, there is a cyclepath graph $G^{\prime} \in \mathcal{U}(n)$ such that $H_{A}(G)>H_{A}\left(G^{\prime}\right)$.

Proof. Let $w$ be a branching vertex on $C$, let $T$ be the connected component of $G \backslash C$ containing $w$, let $x \in T$ be a branching vertex in $T$ furthest from $w$. If $x=w$ let $k=\delta_{G}(x)-2$, else if $x \neq w$ let $k=\delta_{G}(x)-1$. For $i=1, \ldots, k$, let $P_{i}=x y_{i} \ldots z_{i}$ be a path in $T$ starting in $x$ such that $d(w, x)<d\left(w, y_{i}\right)$. Since $x$ is the branching 


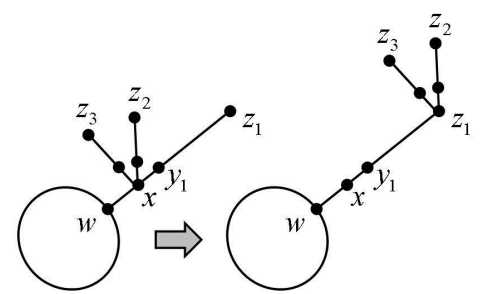

FIGURE 6. Graph transformation from the proof of Lemma 7.

vertex furthest from $w$, these paths are obviously vertex disjoint except for the vertex $x$. Without loss of generality we may assume that $P_{1}$ is the longest among these paths. Let $G^{\prime}$ be a graph obtained from $G$ by deleting edges $x y_{i}$ for $i=2, \ldots, k-1$, and adding edges $z_{1} y_{i}$ instead. This transformation is illustrated in Figure 6. Note that $G^{\prime}$ is a unicyclic graph. We have to prove that $H_{A}(G)>H_{A}\left(G^{\prime}\right)$. Since the value of the index $H_{A}$ has to decrease, the problem are the pairs of vertices whose distance decreases or vertices whose degree increases. Distances possibly decrease only for pairs $u, v$ where $u \in P_{i}(i=2, \ldots, k)$ and $v \in P_{1}$. The only vertex for which the degree increases in this transformation is $z_{1}$.

Let us first consider the problem with the decrease in distances. Let $v \mapsto v^{\prime}$ be the automorphism of the path $P_{1}$ such that $x^{\prime}=z_{1}$. For $u \in P_{i \geq 2}$ we have

$$
\Delta(u, x)+\Delta\left(u, z_{1}\right)=\frac{1}{d_{G}(u, x)}-\frac{1}{d_{G}(u, x)+\left|P_{1}\right|}>0 .
$$

For $u \in P_{i \geq 2}$ and $v \in P_{1} \backslash\left\{x, z_{1}\right\}$, if $v=v^{\prime}$ then $d_{G}(u, v)=d_{G^{\prime}}(u, v)$ so $\Delta(u, v)=0$, else if $v \neq v^{\prime}$ then we have $d_{G}(x, v)=d_{G}\left(z, v^{\prime}\right)$ and $d_{G}(z, v)=d_{G}\left(x, v^{\prime}\right)$ so it is easily verified that $\Delta(u, v)+\Delta\left(u, v^{\prime}\right)=0$.

Let us now consider the problem with the increase in degree. Let again $v \mapsto v^{\prime}$ be the automorphism of path $P_{1}$ such that $x^{\prime}=z_{1}$. For $v \in P_{i}$ we have already considered and compensated negative contributions $\Delta\left(z_{1}, v\right)$. If we now consider $v \in P_{1}$, then for $v=x$ we have

$$
\Delta\left(z_{1}, x\right)=\frac{1+\delta_{G}(x)}{d_{G}\left(z_{1}, x\right)}-\frac{(1+k-1)+\left(\delta_{G}(x)-k+1\right)}{d_{G}\left(z_{1}, x\right)}=0,
$$

while for $v \in P_{1} \backslash\left\{z_{1}, x\right\}$, we have

$$
\Delta\left(z_{1}, v\right)+\Delta\left(x, v^{\prime}\right)=\frac{\delta_{G}(x)-2}{d_{G}\left(x, v^{\prime}\right)}-\frac{\delta_{G}(x)-2}{d_{G}\left(z_{1}, v\right)}=\left[d_{G}\left(z_{1}, v\right)=d_{G}\left(x, v^{\prime}\right)\right]=0 .
$$

Finally, we have to consider $v \in G \backslash\left(\cup_{i=1}^{k} P_{i}\right)$. We have

$$
\Delta\left(z_{1}, v\right)+\Delta(x, v)=\frac{k-1}{d_{G}(x, v)}-\frac{k-1}{d_{G}\left(z_{1}, v\right)}>\left[d_{G}(x, v)<d_{G}\left(z_{1}, v\right)\right]>0 .
$$


Therefore, we have proved that $H_{A}(G)>H_{A}\left(G^{\prime}\right)$. If $G^{\prime}$ is a cycle-path graph, then the proof is completed. If not, then by repeating this transformation finitely many times we obtain a cycle path graph $G^{\prime}$ for which $H_{A}(G)>H_{A}\left(G^{\prime}\right)$, so the proof is complete.

a)

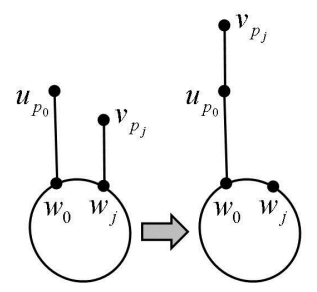

b)

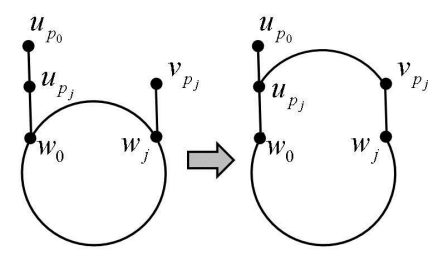

FIGURE 7. Graph transformation from the proof of Lemma 8: a) case $1, b$ ) case $2 a$.

Lemma 8. Let $G \in U(n)$ be a cycle-path graph with at least 2 branching vertices. Then there is a cycle-path graph $G^{\prime} \in \mathcal{U}(n)$ with only one branching vertex such that $H_{A}(G)>H_{A}\left(G^{\prime}\right)$.

Proof. Let $C$ be the only cycle in $G$ with vertices denoted by $w_{i}$. Let $P_{i}$ be the path appended to the branching vertex $w_{i} \in C$ (here $P_{i}$ includes $w_{i}$ ) and let $p_{i}=\left|P_{i}\right|$. We distinguish several cases.

CASE 1. There are two consecutive branching vertices $w_{i}, w_{j}$ on $C$ such that $d\left(w_{i}, w_{j}\right) \leq p_{i}$ or $d\left(w_{i}, w_{j}\right) \leq p_{j}$.

Without loss of generality we may assume that $0=i<j \leq\left\lfloor\frac{c}{2}\right\rfloor, d\left(w_{0}, w_{j}\right) \leq$ $p_{0}$ and $p_{0} \geq p_{j}$. Let $P_{0}=w_{0} u_{1} \ldots u_{p_{0}}$ and $P_{j}=w_{j} v_{1} \ldots v_{p_{j}}$. Let $G^{\prime}$ be a graph obtained from $G$ by deleting the edge $w_{j} v_{1}$ and adding the edge $u_{p_{0}} v_{1}$ instead. This transformation is illustrated in Figure 7 a). Note that $G^{\prime}$ is a cycle-path graph with one branch less than $G$. We have to prove $H_{A}(G)>H_{A}\left(G^{\prime}\right)$. Let $P_{A}$ be the shortest path in $G$ connecting vertices $u_{p_{0}}$ and $v_{p_{j}}$. Note that in this transformation distances possibly decrease only for pairs of vertices $u, v \in P_{A}$. The only vertex for which the degree increases is $u_{p_{0}}$.

Let us first consider the problem with distances. Let $P_{A}^{\prime}$ be a path in $G^{\prime}$ connecting vertices $v_{p_{j}}$ and $w_{j}$. Let $v \mapsto v^{\prime}$ be an isomorphism of paths $P_{A}$ and $P_{A}^{\prime}$ such that $v_{p_{j}}^{\prime}=v_{p_{j}}$. Note that $u_{p_{0}}^{\prime}=w_{j}$ (and vice versa) and also $w_{0}^{\prime} \in P_{0}$ since $d\left(w_{0}, w_{j}\right) \leq$ $p_{0}$. Now, we first consider $u=v_{a} \in P_{j}$. If $v=v_{b} \in P_{j}$ we have $\Delta\left(v_{a}, v_{b}\right)=0$, else if $v=w_{j}$ and $v^{\prime}=u_{p_{0}}$ we have

$$
\Delta\left(v_{a}, w_{j}\right)+\Delta\left(v_{a}, u_{p_{0}}\right)=\frac{1}{d_{G}\left(v_{a}, w_{j}\right)}-\frac{1}{d_{G}\left(v_{a}, w_{j}\right)+p_{0}+j}>0,
$$


else if $v=v^{\prime}$ then from $d_{G^{\prime}}\left(v_{a}, v\right)=d_{G}\left(v_{a}, v\right)$ we have $\Delta\left(v_{a}, v\right)=0$, else if $v=w_{0}$ and $v^{\prime}=w_{0}^{\prime}$ we have

$$
\Delta\left(v_{a}, w_{0}\right)+\Delta\left(v_{a}, w_{0}^{\prime}\right)=\frac{1}{d_{G}\left(v_{a}, w_{j}\right)+j}-\frac{1}{d_{G}\left(v_{a}, w_{j}\right)+p_{0}} \geq\left[j \leq p_{0}\right] \geq 0 .
$$

else if $v \neq v^{\prime}$ then from $d_{G}\left(w_{j}, v^{\prime}\right)=d_{G}\left(u_{p_{0}}, v\right)$ and $d_{G}\left(u_{p_{0}}, v^{\prime}\right)=d_{G}\left(w_{j}, v\right)$ we have $\Delta\left(v_{a}, v\right)+\Delta\left(v_{a}, v^{\prime}\right)=0$. Therefore we have covered all pairs $u, v \in P_{A}$ for which $u \in P_{j}$. Let now $u=u_{p_{0}}$. We have already considered pairs with $v \in P_{j} \backslash\left\{w_{j}\right\}$ in previous text. If $v=w_{j}$ we have $\Delta\left(u_{p_{0}}, w_{j}\right)=0$, else

$$
\Delta\left(u_{p_{0}}, v\right)+\Delta\left(w_{j}, v^{\prime}\right)=\frac{1}{d_{G}\left(w_{j}, v^{\prime}\right)}-\frac{1}{d_{G}\left(u_{p_{0}}, v\right)}=\left[d_{G}\left(w_{j}, v^{\prime}\right)=d_{G}\left(u_{p_{0}}, v\right)\right]=0
$$

Finally, for $u, v \in P_{A} \backslash\left(P_{j} \cup\left\{u_{p_{0}}, w_{j}\right\}\right)$ both the degrees and the distances have not changed in the transformation, so we have $\Delta(u, v)=0$. Therefore, we have considered and compensated all negative contributions due to decrease in distances.

Let us now consider all negative contributions due to increase in degree of $u_{p_{0}}$. Note that we have already considered and compensated pairs $u_{p_{0}}, v$ where $v \in P_{A}$. Let now $v \in G \backslash P_{A}$. We have

$$
\Delta\left(u_{p_{0}}, v\right)+\Delta\left(w_{j}, v\right)=\frac{1}{d_{G}\left(w_{j}, v\right)}-\frac{1}{d_{G}\left(u_{p_{0}}, v\right)}=\left[d_{G}\left(w_{j}, v\right) \leq d_{G}\left(u_{p_{0}}, v\right)\right] \geq 0 .
$$

CASE 2. For every two consecutive branching vertices $w_{i}, w_{j}$ on $C$ it holds that $d\left(w_{i}, w_{j}\right)>p_{i}$ and $d\left(w_{i}, w_{j}\right)>p_{j}$.

SUBCASE 2a. There are at least 4 branching vertices on $C$.

Let $w_{i}$ and $w_{j}$ be the pair of branching vertices on minimum distance. Without loss of generality we may assume that $0=i<j \leq\left\lfloor\frac{c}{4}\right\rfloor$. Let $P_{0}=w_{0} u_{1} \ldots u_{p_{0}}$ and $P_{j}=w_{j} v_{1} \ldots v_{p_{j}}$ be paths appended to $w_{0}$ and $w_{j}$. The condition of Case 2 is now read as $j>p_{0}$ and $j>p_{j}$. Without loss of generality we may assume $p_{0} \geq p_{j}$. Now, let $G^{\prime}$ be a graph obtained from $G$ by deleting edges $w_{0} w_{1}$ and $w_{j-1} w_{j}$ and adding edges $u_{p_{j}} w_{1}$ and $w_{j-1} v_{p_{j}}$ instead. This transformation is illustrated in Figure $7 \mathrm{~b}$ ). Graph $G^{\prime}$ is obviously a cycle-path graph with one branch less than $G$. We have to prove $H_{A}(G)>H_{A}\left(G^{\prime}\right)$. Let $P_{A}$ be the shortest path in $G$ connecting vertices $u_{p_{0}}$ and $v_{p_{j}}$. Note that distances possibly decrease only for pairs of vertices $u, v \in P_{A}$. The only vertices for which degree increases are $u_{p_{j}}$ and $v_{p_{j}}$.

Let us first consider the problem with distances. Let $P_{B}$ be the shortest path in $G$ connecting vertices $u_{p_{j}}$ and $v_{p_{j}}\left(P_{B}\right.$ is subpath of $\left.P_{A}\right)$. Let $P_{B}^{\prime}$ be the shortest path in $G^{\prime}$ connecting vertices $w_{0}$ and $w_{j}$. It is important to note that because of the condition of subcase it holds that $2 p_{j}+j<3\left\lfloor\frac{c}{4}\right\rfloor$, so the shortest path between $w_{0}$ and $w_{j}$ both before and after the transformation goes through the same side of cycle (i.e. paths $P_{B}$ and $P_{B}^{\prime}$ are of the same length and contain the same vertices though not in the same order). Let $v \mapsto v^{\prime}$ be the isomorphism of paths $P_{B}$ and $P_{B}^{\prime}$ such that $u_{p_{j}}^{\prime}=w_{0}$. We first consider pairs $u, v \in P_{B}$. For pairs $u, v \in P_{B}$ such that $u, v \in\left\{u_{p_{j}}, v_{p_{j}}, w_{0}, w_{j}\right\}$, 
it is easily verified that $\Delta\left(u_{p_{j}}, w_{j}\right)=\Delta\left(v_{p_{j}}, w_{0}\right)=\Delta\left(u_{p_{j}}, w_{0}\right)=\Delta\left(v_{p_{j}}, w_{j}\right)=0$ and

$$
\Delta\left(u_{p_{j}}, v_{p_{j}}\right)+\Delta\left(w_{0}, w_{j}\right)=\frac{3-\delta_{G}\left(u_{p_{j}}\right)}{j}-\frac{3-\delta_{G}\left(u_{p_{j}}\right)}{2 p_{j}+j}>0 .
$$

Now, of the remaining pairs $u, v \in P_{B}$, let us consider pairs where $u \in P_{0}$. First, let $u=u_{p_{j}} \in P_{0}$. Then, for $v=u_{b} \in P_{0}$ and $v=v_{b} \in P_{j}\left(b<p_{j}\right)$ we have

$$
\begin{aligned}
\Delta\left(u_{p_{j}}, u_{b}\right)+\Delta\left(w_{0}, u_{b}^{\prime}\right) & =\frac{1}{d_{G}\left(w_{0}, u_{b}^{\prime}\right)}-\frac{1}{d_{G}\left(u_{p_{j}}, u_{b}\right)} \\
& =\left[d_{G}\left(u_{p_{j}}, u_{b}\right)=d_{G}\left(w_{0}, u_{b}^{\prime}\right)\right]=0, \\
\Delta\left(u_{p_{j}}, v_{b}\right)+\Delta\left(w_{0}, v_{b}^{\prime}\right) & =\frac{2-\delta_{G}\left(u_{p_{j}}\right)}{j+p_{j}-b}-\frac{2-\delta_{G}\left(u_{p_{j}}\right)}{p_{j}+j+b}>0,
\end{aligned}
$$

and for $v=w_{b} \in C(0<b<j)$ we have

$$
\Delta\left(u_{p_{j}}, w_{b}\right)+\Delta\left(w_{0}, w_{b}\right)=\frac{2-\delta_{G}\left(u_{p_{j}}\right)}{b}-\frac{2-\delta_{G}\left(u_{p_{j}}\right)}{p_{j}+b}>0 .
$$

Now, let $u=u_{a} \in P_{0}$ for $a<p_{j}$. Then for $b<p_{j}$, if $u_{a}=u_{a}^{\prime}$ and $v_{b}=v_{b}^{\prime}$ (i.e. $a=b=\frac{p_{j}}{2}$ ) we have $\Delta\left(u_{a}, v_{b}\right)=0$, else we have $\Delta\left(u_{a}, v_{b}\right)+\Delta\left(u_{a}^{\prime}, v_{b}^{\prime}\right)=0$. Also, for $0<b<j$, if $u_{a}=u_{a}^{\prime}$ (i.e. $a=\frac{p_{j}}{2}$ ) then $\Delta\left(u_{a}, w_{b}\right)=0$, else $\Delta\left(u_{a}, w_{b}\right)+$ $\Delta\left(u_{a}^{\prime}, w_{b}\right)=0$. Completely analogously one can obtain analogous results for pairs $u, v \in P_{B}$ where $u \in P_{j}$. Now, for all unconsidered pairs $u, v \in P_{B}$ neither degrees change, nor the distance, therefore for those pairs it holds that $\Delta(u, v)=0$. Hence, we have considered all pairs $u, v \in P_{B}$.

It remains to consider pairs $u, v \in P_{A}$ where $u \in P_{A} \backslash P_{B}$. Now, let $u=u_{p_{j}+a} \in$ $P_{A} \backslash P_{B}$. If $v \in P_{A} \backslash P_{B}$ then obviously $\Delta(u, v)=0$, else if $v \in\left\{w_{0}, u_{p_{j}}\right\}$ we have

$$
\begin{aligned}
\Delta\left(u_{p_{j}+a}, w_{0}\right)+\Delta\left(u_{p_{j}+a}, u_{p_{j}}\right) & =\frac{1}{p_{j}+a}-\frac{1}{a}<0, \\
\Delta\left(w_{-a}, w_{0}\right)+\Delta\left(w_{-a}, u_{p_{j}}\right) & =\frac{1}{a}-\frac{1}{a+p_{j}}>0,
\end{aligned}
$$

so we obtain $\Delta\left(u_{p_{j}+a}, w_{0}\right)+\Delta\left(u_{p_{j}+a}, u_{p_{j}}\right)+\Delta\left(w_{-a}, w_{0}\right)+\Delta\left(w_{-a}, u_{p_{j}}\right)=0$. Else if $v \in\left\{w_{j}, v_{p_{j}}\right\}$ we have

$$
\begin{aligned}
\Delta\left(u_{p_{j}+a}, w_{j}\right)+\Delta\left(u_{p_{j}+a}, v_{p_{j}}\right) & =\frac{1}{j+a+p_{j}}-\left(\frac{\delta_{G}\left(u_{p_{j}+a}\right)+2}{a+j}-\frac{\delta_{G}\left(u_{p_{j}+a}\right)+1}{p_{j}+a+j+p_{j}}\right), \\
\Delta\left(w_{j+a}, w_{0}\right)+\Delta\left(w_{j+a}, u_{p_{j}}\right) & =\left(\frac{5}{a+j}-\frac{4}{j+a+p_{j}+p_{j}}\right)-\frac{1}{j+a+p_{j}},
\end{aligned}
$$

so we obtain

$$
\Delta\left(u_{p_{j}+a}, w_{j}\right)+\Delta\left(u_{p_{j}+a}, v_{p_{j}}\right)+\Delta\left(w_{j+a}, w_{0}\right)+\Delta\left(w_{j+a}, u_{p_{j}}\right)
$$




$$
=\frac{3-\delta_{G}\left(u_{p_{j}+a}\right)}{a+j}-\frac{3-\delta_{G}\left(u_{p_{j}+a}\right)}{j+a+p_{j}+p_{j}}>0 .
$$

Else if $v \in\left\{u_{0}, \ldots, u_{p_{j}-1}\right\}$ we have $\Delta\left(u_{p_{j}+a}, v\right)=0$. Else if $v \in\left\{w_{1}, \ldots, w_{j-1}\right\}$ we have

$$
\Delta\left(u_{p_{j}+a}, w_{b}\right)+\Delta\left(w_{-a}, w_{b}\right)=\frac{2-\delta_{G}\left(u_{p_{j}+a}\right)}{a+b}-\frac{2-\delta_{G}\left(u_{p_{j}+a}\right)}{a+b+p_{j}}>0 .
$$

Else if $v \in\left\{v_{1}, \ldots, v_{p_{j}-1}\right\}$ we have

$$
\Delta\left(u_{p_{j}+a}, v_{b}\right)+\Delta\left(w_{-a}, v_{b}^{\prime}\right)=\frac{2-\delta_{G}\left(u_{p_{j}+a}\right)}{a+j+p_{j}-b}-\frac{2-\delta_{G}\left(u_{p_{j}+a}\right)}{a+p_{j}+j+b}>0 .
$$

Therefore, we have considered and compensated all negative contributions due to decrease in distances.

Let us now consider all negative contributions due to increase in degree of $u_{p_{j}}$ and $v_{p_{j}}$. First, note that we have already considered pairs $u_{p_{j}}, v$ and $v_{p_{j}}, v$ for which $v \in$ $P_{A}$. For $u=u_{p_{j}}$ we have also already considered pairs where $v=w_{-a}$ or $v=w_{j+a}$ $\left(a=1, \ldots, p_{0}-p_{j}\right)$. Now, let $v$ be an unconsidered vertex, then from $d_{G}\left(w_{0}, v\right) \leq$ $d_{G^{\prime}}\left(w_{0}, v\right)$ and $d_{G}\left(u_{p_{j}}, v\right)=d_{G^{\prime}}\left(u_{p_{j}}, v\right)$ we have

$$
\Delta\left(u_{p_{j}}, v\right)+\Delta\left(w_{0}, v\right) \geq \frac{1}{d_{G}\left(w_{0}, v\right)}-\frac{1}{d_{G}\left(u_{p_{j}}, v\right)}>\left[d_{G}\left(w_{0}, v\right)<d_{G}\left(u_{p_{j}}, v\right)\right]>0 .
$$

Completely analogously one obtains $\Delta\left(v_{p_{j}}, v\right)+\Delta\left(w_{j}, v\right)>0$ and the proof of this subcase is over.

SUBCASE $2 \mathrm{~b}$. There are exactly 3 branching vertices on $C$.

Let $w_{0}, w_{j}$ and $w_{k}$ be three branching vertices on $C$. Let us denote $d_{1}=d_{G}\left(w_{0}, w_{j}\right), d_{2}=d_{G}\left(w_{j}, w_{k}\right), d_{3}=d_{G}\left(w_{k}, w_{0}\right)$. Without loss of generality we may assume that $d_{3} \geq \max \left\{d_{1}, d_{2}\right\}$. We will also need $d_{4}=\min \left\{d_{1}+d_{2}, d_{3}\right\} \geq$ $d_{2}$. Let $P_{0}=w_{0} u_{1} \ldots u_{p_{0}}, P_{j}=w_{j} v_{1} \ldots v_{p_{j}}, P_{k}=w_{k} z_{1} \ldots z_{p_{k}}$ be paths appended to branching vertices on $C$. Without loss of generality we may assume $p_{0} \leq p_{k}$. Let $G^{\prime}$ be the graph obtained from $G$ by deleting edge $w_{j} v_{1}$ and adding the edge $u_{p_{0}} v_{1}$ instead. Note that $G^{\prime}$ is a cycle-path graph with one branch less than $G$. We have to prove $H_{A}(G)>H_{A}\left(G^{\prime}\right)$. Note that distances possibly decrease only for pairs $u, v$ for which $u \in P_{j}$ and $v \in G$. The only vertex whose degree increases is $u_{p_{0}}$.

We will first consider the problem with distances, so let $u=v_{a} \in P_{j}$. Let $P_{A}$ be the shortest path in $G$ connecting vertices $v_{p_{j}}$ and $u_{p_{0}}$, let $P_{A}^{\prime}$ be the shortest path in $G^{\prime}$ connecting vertices $v_{p_{j}}$ and $w_{j}$. Let $v \mapsto v^{\prime}$ be an isomorphism of paths $P_{A}$ and $P_{A}^{\prime}$ such that $v_{p_{j}}=v_{p_{j}}^{\prime}$. Note that $w_{0}^{\prime} \in\left\{w_{1}, \ldots, w_{j-1}\right\}$ since $p_{0}<j$ (supposition of Case 2). First, we will consider cases where $v \in P_{A}$. If $v=v_{b} \in P_{j}$, then we have $\Delta\left(v_{a}, v_{b}\right)=0$. Else if $v \in\left\{w_{j}, u_{p_{0}}, w_{0}^{\prime}, w_{0}\right\}$ we have

$$
\Delta\left(v_{a}, u_{p_{0}}\right)+\Delta\left(v_{a}, w_{j}\right)=\frac{1}{a}-\frac{1}{a+d_{1}+p_{0}}>0,
$$




$$
\Delta\left(v_{a}, w_{0}\right)+\Delta\left(v_{a}, w_{0}^{\prime}\right)=\frac{1}{a+d_{1}}-\frac{1}{a+p_{0}}<\left[d_{1}>p_{0}\right]<0,
$$

so obviously

$$
\begin{aligned}
\Delta\left(v_{a}, u_{p_{0}}\right)+\Delta\left(v_{a}, w_{j}\right)+\Delta\left(v_{a}, w_{0}\right) & +\Delta\left(v_{a}, w_{0}^{\prime}\right) \\
= & \frac{1}{a}-\frac{1}{a+p_{0}}+\frac{1}{a+d_{1}}-\frac{1}{a+d_{1}+p_{0}}>0 .
\end{aligned}
$$

Else if $v=v^{\prime}$ from $d_{G}\left(w_{j}, v\right)=d_{G}\left(u_{p_{0}}, v\right)$ we have $\Delta\left(v_{a}, v\right)=0$. Else if $v \neq$ $v^{\prime}$ from $d_{G}\left(v_{a}, v^{\prime}\right)=d_{G^{\prime}}\left(v_{a}, v\right)$ and $d_{G^{\prime}}\left(v_{a}, v^{\prime}\right)=d_{G}\left(v_{a}, v\right)$ we have $\Delta\left(v_{a}, v\right)+$ $\Delta\left(v_{a}, v^{\prime}\right)=0$. Therefore, we have considered all cases where $v \in P_{A}$. Let us now consider cases where $v \in C \backslash P_{A}$. Let $v \mapsto v^{\prime}$ be the automorphism of cycle $C$ such that $w_{0}^{\prime}=w_{j}$ and $w_{j}^{\prime}=w_{0}$. If $v=v^{\prime}$ then from $d_{G}\left(w_{j}, v\right)=d_{G}\left(u_{p_{0}}, v\right)$ we have

$$
\Delta\left(v_{a}, v\right)=\frac{\delta_{G}\left(v_{a}\right)+\delta_{G}(v)}{d_{G}\left(v_{a}, w_{j}\right)+d_{G}\left(w_{j}, v\right)}-\frac{\delta_{G}\left(v_{a}\right)+\delta_{G}(v)}{d_{G}\left(v_{a}, w_{j}\right)+p_{0}+d_{G}\left(w_{j}, v\right)}>0,
$$

else if $v \in\left\{w_{k}, w_{k}^{\prime}\right\}$ we have

$$
\begin{aligned}
\Delta\left(v_{a}, w_{k}\right)+\Delta\left(v_{a}, w_{k}^{\prime}\right) & =\frac{\delta_{G}\left(v_{a}\right)+3}{a+d_{2}}-\frac{\delta_{G}\left(v_{a}\right)+3}{a+p_{0}+d_{4}}+\frac{\delta_{G}\left(v_{a}\right)+2}{a+d_{4}}-\frac{\delta_{G}\left(v_{a}\right)+2}{a+p_{0}+d_{2}} \\
& >\left[\frac{\delta_{G}\left(v_{a}\right)+3}{a+d_{4}}-\frac{\delta_{G}\left(v_{a}\right)+3}{a+p_{0}+d_{4}}>0\right] \\
& >\frac{\delta_{G}\left(v_{a}\right)+3}{a+d_{2}}-\frac{\delta_{G}\left(v_{a}\right)+2}{a+p_{0}+d_{2}}-\frac{1}{a+d_{4}} \geq\left[d_{4} \geq d_{2}\right] \\
& \geq \frac{\delta_{G}\left(v_{a}\right)+2}{a+d_{2}}-\frac{\delta_{G}\left(v_{a}\right)+2}{a+p_{0}+d_{2}}>0,
\end{aligned}
$$

else from $d_{G}\left(w_{j}, v^{\prime}\right)=d_{G}\left(w_{0}, v\right)$ and $d_{G}\left(w_{0}, v^{\prime}\right)=d_{G}\left(w_{j}, v\right)$ we have

$$
\begin{aligned}
\Delta\left(v_{a}, v\right)+\Delta\left(v_{a}, v^{\prime}\right)= & \frac{\delta_{G}\left(v_{a}\right)+2}{a+d_{G}\left(w_{0}, v\right)}-\frac{\delta_{G}\left(v_{a}\right)+2}{a+p_{0}+d_{G}\left(w_{0}, v\right)}+ \\
& +\frac{\delta_{G}\left(v_{a}\right)+2}{a+d_{G}\left(w_{j}, v\right)}-\frac{\delta_{G}\left(v_{a}\right)+2}{a+p_{0}+d_{G}\left(w_{j}, v\right)}>0 .
\end{aligned}
$$

Finally, we have to consider $v=z_{b} \in P_{k}$. We have

$$
\Delta\left(v_{a}, z_{b}\right)=\frac{\delta_{G}\left(v_{a}\right)+\delta_{G}\left(z_{b}\right)}{a+d_{2}+b}-\frac{\delta_{G}\left(v_{a}\right)+\delta_{G}\left(z_{b}\right)}{a+d_{4}+b} \geq\left[d_{2} \leq d_{4}\right] \geq 0 .
$$

Therefore, we have considered and compensated all negative contributions due to decrease in distances.

Let us now consider all negative contributions due to increase in degree of $u_{p_{0}}$, so let $u=u_{p_{0}}$. We have already considered pairs $u, v$ where $v \in P_{j} \backslash\left\{w_{j}\right\}$. For the 
remaining possibilities for $v$, we will again use already introduced isomorphisms of $P_{A}$ and $C$. If $v=w_{j}$ then $\Delta\left(u_{p_{0}}, w_{j}\right)=0$, else if $v \in P_{A} \backslash P_{j}$ we have

$$
\Delta\left(u_{p_{0}}, v\right)+\Delta\left(w_{j}, v^{\prime}\right)=\frac{1}{d_{G}\left(w_{j}, v^{\prime}\right)}-\frac{1}{d_{G}\left(u_{p_{0}}, v\right)}=\left[d_{G}\left(u_{p_{0}}, v\right)=d_{G}\left(w_{j}, v^{\prime}\right)\right]=0,
$$

else if $v \in C \backslash P_{A}$ we have

$$
\Delta\left(u_{p_{0}}, v\right)+\Delta\left(w_{j}, v^{\prime}\right)=\frac{1}{d_{G}\left(w_{0}, v^{\prime}\right)}-\frac{1}{d_{G}\left(u_{p_{0}}, v\right)}=\left[d_{G}\left(w_{0}, v^{\prime}\right)=d_{G}\left(u_{p_{0}}, v\right)\right]=0,
$$

else if $v=z_{a} \in P_{k} \backslash\left\{w_{k}\right\}$ we have

$$
\Delta\left(u_{p_{0}}, z_{a}\right)+\Delta\left(w_{j}, z_{a}\right)=\frac{1}{d_{2}+a}-\frac{1}{p_{0}+d_{4}+a}>0 .
$$

SUBCASE 2c. There are exactly 2 branching vertices on $C$.

Let $w_{0}$ and $w_{j}$ be branching vertices on $C$ and let $P_{0}=w_{0} u_{1} \ldots u_{p_{0}}, P_{j}=$ $w_{j} v_{1} \ldots v_{p_{j}}$ be paths appended to branching vertices. Let $G^{\prime}$ be a graph obtained from $G$ by deleting the edge $w_{j} v_{1}$ and adding the edge $u_{p_{0}} v_{1}$ instead. Graph $G^{\prime}$ is obviously a cycle-path graph with only one branch. Proof that $H_{A}(G)>H_{A}\left(G^{\prime}\right)$ is completely analogous to the proof of subcase $2 b$, one just doesn't have to consider vertex $w_{k}$ separately and there are no vertices $z_{a}$.

So, in all cases we have proved $H_{A}(G)>H_{A}\left(G^{\prime}\right)$. Since in all cases $G^{\prime}$ is a cyclepath graph with one branch less than in $G$, we have either obtained a cycle-path graph $G^{\prime}$ which has only one branch, or by repeating the transformation finitely many times we will obtain such graph. Therefore, the lemma is proved.

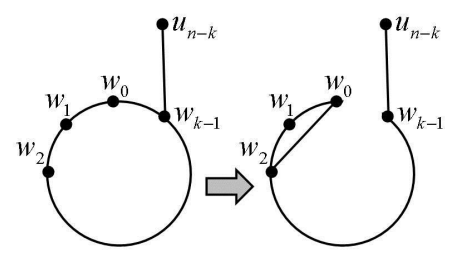

FIGURE 8. Graph transformation from the proof of Lemma 9.

Lemma 9. Let $G=C_{k, n-k}$ where $k \geq 4$ and $n \geq 5$. Then $H_{A}(G)>H_{A}\left(C_{3, n-3}\right)$.

Proof. Let us denote vertices in $G$ so that for the only cycle in $G$ holds $C=$ $w_{0} w_{1} \ldots w_{k-1}$ where $4 \leq k \leq n$. If there is a branching vertex in $G$, without loss of generality we may assume that it is $w_{k-1}$ and that $P_{k-1}=w_{k-1} u_{1} \ldots u_{n-k}$ is the only path appended to $w_{k-1}$. Let $G^{\prime}$ be a graph obtained from $G$ by deleting the edge $w_{0} w_{k-1}$ and adding the edge $w_{0} w_{2}$ instead. This transformation is illustrated in Figure 8. Note that $G^{\prime}=C P_{3, n-3}$. We have to prove $H_{A}(G)>H_{A}\left(G^{\prime}\right)$. Note that 
distances in this transformation decrease only for $u, v$ where $u=w_{0}$ and $v=w_{i}$ ( $i=$ $2, \ldots,\lfloor k / 2\rfloor)$. The only vertex whose degree increases is $w_{2}$. For the ease of proving the lemma, we introduce $d_{m}=\min \{3, k-3\}$ and the path $P_{A}=w_{2} w_{3} \ldots w_{k-1}$ in $G$ with the automorphism $v \mapsto v^{\prime}$ of $P_{A}$ such that $w_{2}^{\prime}=w_{k-1}$. Now, we distinguish two cases with respect to whether $G=C_{n}(k=n)$ or $G=G=C_{k, n-k}(4 \leq k \leq n-1)$.

CASE 1. Let $G=C_{n}(k=n)$. We will first consider the problem with distances. Let $u=w_{0}$. If $v=w_{2}$ we have

$$
\Delta\left(w_{0}, w_{2}\right)+\Delta\left(w_{0}, w_{k-1}\right)=\frac{1}{2} \frac{7 k-20}{k-2}>0,
$$

else if $v=w_{i}(i=3, \ldots,\lfloor k / 2\rfloor)$ we have

$$
\Delta\left(w_{0}, w_{i}\right)+\Delta\left(w_{0}, w_{k-i+1}\right)=\frac{4}{i}-\frac{4}{k-i} \geq[i \leq k-i] \geq 0 .
$$

Let us now consider the problem with the increase in degree of $w_{2}$, so let $u=w_{2}$. Note that we have already considered pairs $u, v$ where $v=w_{0}$. If $v=w_{1}$ we have

$$
\Delta\left(w_{2}, w_{1}\right)+\Delta\left(w_{k-1}, w_{1}\right)=\frac{k-5}{k-2} \geq[k=n \geq 5] \geq 0,
$$

else if $v=w_{k-1}$ we have

$$
\Delta\left(w_{2}, w_{k-1}\right)=\frac{2+2}{d_{m}}-\frac{3+1}{k-3} \geq\left[k-3 \leq d_{m}\right] \geq 0,
$$

else using the automorphism of $P_{A}$ (and supposing $d_{P_{A}}\left(w_{2}, v\right) \leq d_{P_{A}}\left(w_{2}, v^{\prime}\right)$ ) from $d_{G}\left(w_{2}, v\right)=d_{G}\left(w_{k-1}, v^{\prime}\right)$ we have $\Delta\left(w_{2}, v\right)+\Delta\left(w_{k-1}, v^{\prime}\right)=0$.

CASE 2. Let $G=C_{k, n-k}$. Again, we first consider the problem with distances. Let $u=w_{0}$, we have to consider $v=w_{2}$ and $v \in\left\{w_{3}, \ldots, w_{\lfloor k / 2\rfloor}\right\}$. We have

$$
\begin{aligned}
\Delta\left(w_{0}, w_{2}\right)+\Delta\left(w_{0}, w_{k-1}\right) & =\frac{2 k-8}{k-2} \geq[k \geq 4] \geq 0 \\
\Delta\left(w_{0}, w_{i}\right)+\Delta\left(w_{0}, w_{k-i+1}\right) & =\frac{4}{i}-\frac{4}{k-i} \geq[i \leq k-i] \geq 0 .
\end{aligned}
$$

Now, let us consider the problem with the increase in degree of $w_{2}$, so let $u \in w_{2}$. Note that we have already considered $v=w_{0}$. We have to consider $v=w_{1}, v=$ $w_{k-1}, v \in P_{A} \backslash\left\{w_{1}, w_{k-1}\right\}, v=u_{a} \in P_{k-1}$. We have

$$
\begin{aligned}
\Delta\left(w_{2}, w_{1}\right)+\Delta\left(w_{k-1}, w_{1}\right) & =\frac{1}{2} \frac{3 k-14}{k-2}>0 \text { for } k \geq 5, \\
\Delta\left(w_{2}, w_{k-1}\right) & =\frac{2+3}{d_{m}}-\frac{3+2}{k-3} \geq\left[d_{m} \leq k-3\right] \geq 0 .
\end{aligned}
$$

Further, for $v \in P_{A} \backslash\left\{w_{1}, w_{k-1}\right\}$, assuming $d_{P_{A}}\left(w_{v}, v\right) \leq d_{P_{A}}\left(w_{v}, v^{\prime}\right)$, we have $\Delta\left(w_{2}, v\right)+\Delta\left(w_{k-1}, v^{\prime}\right)=0$. Finally, using $d_{m} \leq k-3$ we obtain

$$
\Delta\left(w_{2}, u_{a}\right)+\Delta\left(w_{k-1}, u_{a}\right) \geq \frac{1}{a}-\frac{1}{d_{m}+a}>0 .
$$


Therefore, the only problem is $\Delta\left(w_{2}, w_{1}\right)+\Delta\left(w_{k-1}, w_{1}\right)$ for $k=4$. But note that in that case $d_{m}=\min \{4,1\}=1$, so we have

$$
\begin{aligned}
& \Delta\left(w_{2}, w_{1}\right)+\Delta\left(w_{k-1}, w_{1}\right)+\Delta\left(w_{2}, u_{1}\right)+\Delta\left(w_{k-1}, u_{1}\right) \\
& =\frac{1}{2} \frac{12-14}{4-2}+\frac{1}{1}-\frac{1}{1+1}=0 .
\end{aligned}
$$

Note that in this case we have not proved the strict inequality if there is only one $u_{a}$, i.e. if $n=5$. But in that case it is easily verified that $H_{A}\left(C_{4,1}\right)>H_{A}\left(C_{3,2}\right)$. The positive contribution which makes the difference is $\Delta\left(w_{0}, u_{a}\right)$ which was not considered in the proof.

Note that $C_{4}$ and $C_{3,1}$ are the only unicyclic graphs on $n=4$ vertices. It holds that

$$
H_{A}\left(C_{4}\right)=20<21=H_{A}\left(C_{3,1}\right) \text {. }
$$

So, for $n=4$ graph $C_{4}$ is the only minimal unicyclic graph, while for $n \geq 5$ the answer to the question of minimal unicyclic graph is given by the following theorem.

Theorem 2. Let $G \in \mathcal{U}(n)$ for $n \geq 5$. Then

$$
H_{A}(G) \geq 4 \sum_{i=1}^{n-2} H_{n-i-1}+H_{n-3}+3 H_{n-2}+\frac{6 n-13}{n-2}
$$

with equality if and only if $G=C P_{3, n-3}$.

Proof. Using Lemmas 7, 8 and 9 we first transform a unicyclic graph to cycle-path graph, then we decrease the number of branches in obtained cycle-path graph, so that finally we can transform it to $C P_{3, n-3}$. In each of these transformations the value of $H_{A}$ strictly decreases, so $\mathrm{CP}_{3, n-3}$ is the only extremal graph. Now, the bound follows from Lemma 1 . Note that the case of $C_{n}$ is covered by Lemma 9 .

\section{CONCLUSION}

In this paper we defined cycle-star graph $C S_{k, n-k}$ to be a graph consisting of cycle of length $k$ and $n-k$ leafs appended to the same vertex of the cycle. Also, we defined cycle-path graph to be a graph consisting of cycle of length $k$ and of path on $n-k$ vertices whose one end is linked to a vertex on a cycle. We establish that $C S_{3, n-3}$ is the only maximal unicyclic graph (see Theorem 1), while $C P_{3, n-3}$ is the only minimal unicyclic graph (see Theorem 2), with respect to additively weighted Harary index. The values of additively weighted Harary index of $C S_{3, n-3}$ and $C P_{3, n-3}$ are established in Lemma 1, so these values are the upper and the lower bound for the value of Harary index on the class of unicyclic graphs. For further research it would be interesting to investigate the values of Harary index on classes of graphs with given parameters, the relation of this variant of Harary index with other topological indices and similar. 


\section{ACKNOWLEDGEMENTS}

The support of the EUROCORES Programme EUROGIGA (project GReGAS) of the European Science Foundation and Croatian Science Foundation under the project 8481 is gratefully acknowledged.

\section{REFERENCES}

[1] Y. Alizadeh, A. Iranmanesh, and T. Doslic, "Additively weighted harary index of some composite graphs," Discret. Math., vol. 313, no. 1, pp. 26-34, 2013, doi: 10.1016/j.disc.2012.09.011.

[2] F. Bruckler, T. Doslic, A. Graovac, and I. Gutman, "On a class of distance-based molecular structure descriptors," Chem. Phys. Lett., vol. 503, pp. 336-338, 2011, doi: 10.1016/j.cplett.2011.01.033.

[3] A. Dobrynin, R. Entringer, and I. Gutman, "Wiener index of trees: theory and applications," Acta Appl. Math., vol. 66, pp. 211-249, 2001, doi: 10.1023/A:1010767517079.

[4] A. Dobrynin, I. Gutman, S. Klavzar, and P. Zigert, "Wiener index of hexagonal systems," Acta Appl. Math., vol. 72, pp. 247-294, 2002, doi: 10.1023/A:1016290123303.

[5] A. Ilic, G. Yu, and L. Feng, "The harary index of trees," Util. Math., vol. 87, pp. 21-31, 2012.

[6] O. Ivanciuc, T. Balaban, and A. Balaban, "Design of topological indices, part 4, reciprocal distance matrix, related local vertex invariants and topological indices," J. Math. Chem., vol. 12, pp. 309318, 1993, doi: 10.1007/BF01164642.

[7] S. Nikolic, N. Trinajstic, and Z. Mihalic, "The wiener index: development and applications," Croat. Chem. Acta, vol. 68, pp. 105-129, 1995.

[8] D. Plavsic, S. Nikolic, and N. Trinajstic, "On the harary index for the characterization of chemical graphs," J. Math. Chem., vol. 12, pp. 235-250, 1993, doi: 10.1007/BF01164638.

[9] S. Wagner, H. Wang, and X. Zhang, "Distance-based graph invariants of trees and the harary index," Filomat, vol. 27, no. 1, pp. 41-50, 2013, doi: 10.2298/FIL1301041W.

[10] H. Wiener, "Structural determination of the paraffin boiling points," J. Am. Chem. Soc., vol. 69, pp. 17-20, 1947, doi: 10.1021/ja01193a005.

[11] K. Xu and K. C. Das, "Extremal unicyclic and bicyclic graphs with respect to harary index," Bull. Malays. Math. Sci. Soc., vol. 36, pp. 373-383, 2013.

[12] B. Zhou, X. Cai, and N. Trinajstic, "On harary index," J. Math. Chem., vol. 44, pp. 611-618, 2008, doi: 10.1007/s10910-007-9339-2.

Author's address

Jelena Sedlar

University of Split, Faculty of civil engineering, architecture and geodesy, Matice hrvatske 15, 21000 Split, Croatia

E-mail address: jsedlaregradst.hr 